Algebra Univers. 69 (2013) 387-399

DOI 10.1007/s00012-013-0236-1

Published online May 9, 2013

(C) 2013 Springer Basel

Algebra Universalis

\title{
Very many clones above the unary clone
}

\author{
Martin Goldstern, Gábor Sági, and Saharon Shelah
}

\begin{abstract}
Let $\mathfrak{c}:=2^{\aleph_{0}}$. We give a family of pairwise incomparable clones on $\mathbb{N}$ with $2^{\mathfrak{c}}$ members, all with the same unary fragment, namely the set of all unary operations.

We also give, for each $n$, a family of $2^{\mathfrak{c}}$ clones all with the same $n$-ary fragment, and all containing the set of all unary operations.
\end{abstract}

\section{Introduction}

In this paper, $X$ will always be a countably infinite set. For a fixed base set $X$, an operation on $X$ is a function $f: X^{n} \rightarrow X$ for some positive natural number $n$. A clone on $X$ is a set of operations that contains all projection functions and is closed under composition. The set of all clones on $X$ ordered by inclusion forms a complete lattice. (The survey paper [3] gives some background about clones, and in particular collects many recent results concerning clones on infinite sets.)

We write $\mathscr{O}^{(n)}$ for the set $X^{X^{n}}$ of all $n$-ary operations. For a clone $C$, call $C^{(n)}:=C \cap \mathscr{O}^{(n)}$ the $n$-ary fragment of $C$. The unary fragment $C^{(1)}$ is a submonoid of the monoid $X^{X}$ of all unary operations. For any monoid $M \subseteq X^{X}$, the set of all clones $C$ with $C^{(1)}=M$ is called the monoidal interval of $M$; it has a least element, the clone generated by $M$, and a largest element $\operatorname{Pol}(M)$, the set of all operations $f$ satisfying $f\left(m_{1}, \ldots, m_{k}\right) \in M$ whenever $m_{1}, \ldots, m_{k} \in M$. (Here, $f\left(m_{1}, \ldots, m_{k}\right)$ is the unary operation mapping $x$ to $f\left(m_{1}(x), \ldots, m_{k}(x)\right)$.)

In [2], we showed that on $X=\mathbb{N}$ there are uncountably many clones containing all unary operations (but only two coatoms, see [1], [4]); in other words, the monoidal interval of $X^{X}$ is uncountable. Pinsker in [6] has constructed (on arbitrary infinite base sets $X$ ) different monoids whose monoidal intervals have various sizes, among them also one whose monoidal interval has size $2^{2^{|X|}}$.

We will show here that (for $|X|=\aleph_{0}$ ) the interval associated with the monoid $X^{X}$ has the largest possible size: $2^{\mathfrak{c}}$. We will also construct, for any

Presented by R. Poeschel.

Received February 25, 2012; accepted in final form November 2, 2012.

2010 Mathematics Subject Classification: Primary: 08A40; Secondary: 05C25, 05C65.

Key words and phrases: clones, nonstructure, monoidal interval.

The first author is supported by the Austrian Science Foundation FWF, grant P 22994N18. The second author is supported by Hungarian National Foundation for Scientific Research grant K83726 and by the János Bolyai Research Scholarship of the Hungarian Academy of Sciences. The third author is supported by the German-Israeli Foundation for Scientific Research \& Development Grant No. 963-98.6/2007. Publication 989.

Birkhäuser 
natural number $n \geq 1$, many clones which share their $n$-ary fragment with $2^{\mathrm{c}}$ other clones.

The rest of this paper is organized as follows. In Subsection 1.1, we announce the main results of the paper in a more precise way. Before doing so, we need further technical preparations. In this subsection, we also present some preliminary observations which we will use later. Section 2 , is devoted to the proof of Theorem 1.1: if $X$ is a countably infinite set, then there exist $2^{\mathfrak{c}}$ clones on $X$ such that each of these clones contain all unary operations on $X$. This is the first main result of the paper. Our construction is based on an Erdős type probabilistic argument. For further motivation and intuitive explanation about our method, we refer to the beginning of Section 2. Finally, in Section 3, we prove Theorem 1.3 which we consider the second main result of the paper (for a detailed formulation of Theorem 1.3, we refer to Subsection 1.1 below).

1.1. Main results. The first main result of the paper is as follows.

Theorem 1.1. Let $X=\mathbb{N}$ be countably infinite. Then there are $2^{\mathfrak{c}}$ clones on $X$ containing the monoid of all unary operations.

To generalize the theorem also to larger arities, we need the following technical definition:

Definition 1.2. Let $\alpha \in \mathbb{R}$. An operation $f: X^{d} \rightarrow X$ is defined to be $\alpha$ modest iff for all natural numbers $N$ and all $Y \subseteq X$ of cardinality $N$, the range of $f\left\lceil Y^{d}\right.$ has at most $\alpha N$ elements.

- $f$ is modest iff $f$ is $\alpha$-modest for some $\alpha$.

- We call a clone $C$ modest iff all operations in $C$ are modest.

- We write $\mathscr{M}$ for the set of all modest operations.

Note that $\mathscr{M}$ is a clone (the greatest modest clone) and that all unary operations are modest; in addition, all operations with finite range are modest, as well.

Theorem 1.3. Let $d \geq 1$ and let $C$ be a modest clone on $\mathbb{N}$ containing all $d$-ary operations with range $\{0,1\}$. Then there are $2^{\mathfrak{c}}$ many clones $D$ with $D \cap \mathscr{O}^{(d)}=C \cap \mathscr{O}^{(d)}$.

Taking $d=1$ and $C$ the clone of all essentially unary operations, we get Theorem 1.1 as a special case.

Machida [5] has defined a natural metric on clones: The distance between two clones is $1 / n$, where $n$ is minimal with $C \cap \mathscr{O}^{(n)} \neq D \cap \mathscr{O}^{(n)}$. In this language, Theorem 1.3 says that certain sets of clones can be arbitrarily small from the metric/topological point of view-and still large when measured by cardinality.

Let $F$ be a set of operations. We write $\langle F\rangle$ for the smallest clone containing $F$. If $C$ is a clone, then we may write $\langle F\rangle_{C}$ instead of $\langle F \cup C\rangle$. Similarly, 
for $F=\{f, g, \ldots\}$, we write $\langle f, g, \ldots\rangle_{C}$ instead of $\langle\{f, g, \ldots\}\rangle_{C}$. Note that $f \in\langle F\rangle_{C}$ iff there is a finite subset $F_{0} \subseteq F$ with $f \in\left\langle F_{0}\right\rangle_{C}$.

Both sections of this paper use the following easy fact:

Lemma 1.4. Let $C$ be a clone, and let $\left(f_{i}: i \in I\right)$ be a family of operations which is independent over $C$ (which means that $f_{i} \notin\left\langle f_{j}: j \neq i\right\rangle_{C}$ for all $i \in I)$. For $J \subseteq I$, let $C_{J}=\left\langle f_{i}: i \in J\right\rangle_{C}$.

(a) The map $J \mapsto C_{J}$ is a 1-1 order-preserving map from $\mathfrak{P}(I)$, the power set of $I$, into the interval $\left[C,\left\langle f_{i}: i \in I\right\rangle_{C}\right]$ in the clone lattice (both ordered by inclusion).

(b) If I has cardinality $\kappa$, then $\left\{C_{J}: J \subseteq I\right\}$ contains $2^{\kappa}$ many elements and it is order-isomorphic with $\mathfrak{P}(I)$.

(c) Assume moreover that $\left\{f_{i}: i \in I\right\} \subseteq \operatorname{Pol}\left(C \cap \mathscr{O}^{(d)}\right)$. (Here, $\operatorname{Pol}\left(C \cap \mathscr{O}^{(d)}\right)$ is the set of all operations $f$ with $f\left(c_{1}, \ldots, c_{m}\right) \in C \cap \mathscr{O}^{(d)}$ whenever $c_{1}, \ldots, c_{m} \in C \cap \mathscr{O}^{(d)}$.) Then $C_{J} \cap \mathscr{O}^{(d)}=C \cap \mathscr{O}^{(d)}$ for all $J \subseteq I$.

Proof. (a) and (b) are clear. The assumption of (c) implies

$$
C \subseteq\left\langle f_{i}: i \in I\right\rangle_{C} \subseteq \operatorname{Pol}\left(C \cap \mathscr{O}^{(d)}\right),
$$

and by definition, the clones $C$ and $\operatorname{Pol}\left(C \cap \mathscr{O}^{(d)}\right)$ have the same $d$-ary fragment $D$. Consequently, the $d$-ary fragment of $C_{J}$ is $D$, as well.

\section{Sparse graphs and modest operations}

Definition 2.1. Let $(V, E)$ be a graph (i.e., $E \subseteq[V]^{2}$, where $[V]^{2}$ is the set of 2-element subsets of $V)$. We say that $(V, E)$ is $(k, l)$-sparse iff for every $U \subseteq V$ of size at most $k$, the induced subgraph on $U$ has at most $l$ edges.

We note that there is an ambiguity in the literature about the notion of sparse graphs. Some authors use this name for graphs with low maximum average degree, some others define a graph to be $(k, l)$-sparse iff no subset of $n$ vertices spans more than $k n-l$ edges. Our notion is slightly different from all of these. We also note that by the size of a graph we mean the cardinality of the set of its vertices (and not, as sometimes done in graph theory, the cardinality of the set of its edges).

In order to help the reader, in this paragraph we are providing a brief and informal explanation for the technical details of the rest of this section. In Lemma 2.3 below, we will show that for all $M$, for all large enough $N$, and for all $0<\varepsilon<\frac{1}{2}$, there exist graphs $\mathcal{G}$ on $N$ vertices whose $M$-sized subgraphs are $(k, l)$-sparse for certain $k$ and $l$ (where $M$ is small relative to $N$ ); while at the same time, these $\mathcal{G}$ have "many" edges: the number of their edges is at least $N^{1+\varepsilon}$. Using this lemma, we will be able to construct functions on finite domains having large range, but the range of their restrictions to small sets remains small; for the details see Lemma 2.6. Carefully "gluing together" an infinite sequence of such operations we obtain a set $S$ of operations on $\mathbb{N}$ 
such that $S$ is independent (over $O^{(1)}$, see Lemma 1.4) and has cardinality c. Combining this with Lemma 1.4, the proof of Theorem 1.1 will follow quickly.

Definition 2.2. Let $M, N$ be natural numbers, and $0<\varepsilon<\frac{1}{2}$. We write $M \ll_{\varepsilon} N$ iff $M \cdot N^{2 \varepsilon-1}<1 / 10$.

Lemma 2.3. Let $0<\varepsilon<1 / 2$ and let $1 \leq M \ll_{\varepsilon} N$. Then there is a graph $G=(V, E)$ with $N$ vertices and more than $N^{1+\varepsilon}$ edges that is $(k, 2 k)$-sparse for all $k \leqslant M$.

Proof. We will use an Erdős type probability argument: we will define a suitable probability measure on all graphs on $N$ vertices and then show that the set of graphs not satisfying the conclusion has small measure.

We note that a somewhat stronger form of the lemma follows quickly from the Central Limit Theorem. For completeness, we present an elementary proof.

Let $p:=4 N^{-1+\varepsilon}$ and let $\mu$ be the probability measure on $\{0,1\}$ with $\mu(\{1\})=p$. Fix a set $V$ of $N$ vertices; there are $\frac{N(N-1)}{2}$ potential edges. Via characteristic functions, we identify the set of all graphs on $V$ with the product space $\{0,1\}^{\frac{N(N-1)}{2}}$, equipped with the product probability structure. In order to keep notation simple, the product measure will also be called $\mu$.

In other words, for each potential edge $e$ we flip a weighted coin (independent of all other coin flips) and with probability $p$ we decide to add $e$ to our graph. The expected number of edges is $\frac{N(N-1)}{2} \cdot p \approx 2 N^{1+\varepsilon}$, with variance $\frac{N(N-1)}{2} p(1-p) \approx 2 N^{1+\varepsilon}$. By Chebyshev's inequality, most graphs will have more than $N^{1+\varepsilon}$ edges. More precisely, the measure of the set of graphs with fewer than $N^{1+\varepsilon}$ edges is smaller than

$$
\frac{\frac{N(N-1)}{2} p(1-p)}{\left(\frac{N(N-1)}{2} \cdot p-N^{1+\varepsilon}\right)^{2}} \approx \frac{2 N^{1+\varepsilon}}{\left(N^{1+\varepsilon}\right)^{2}}=2 N^{-1-\varepsilon}<1 / 2,
$$

because, by the assumptions of the lemma, we have $4 \leq N$.

We now estimate the measure of the set $\mathscr{G}$ of all graphs on $V$ which are not $(k, 2 k)$-sparse for some $k \leqslant M$.

For any set $E^{\prime} \subseteq[V]^{2}$, we let $\mathscr{G}_{E^{\prime}}$ be the set of all graphs whose edges include the set $E^{\prime}$. Clearly, $\mu\left(\mathscr{G}_{E^{\prime}}\right)=\left(4 N^{-1+\varepsilon}\right)^{\left|E^{\prime}\right|}$.

For each graph $(V, E)$ which is not $(k, 2 k)$-sparse, there exists a set $V^{\prime}$ of $k$ vertices and a set $E^{\prime} \subseteq\left[V^{\prime}\right]^{2}$ with $2 k$ elements such that $E \supseteq E^{\prime}$, i.e., $(V, E) \in \mathscr{G}_{E^{\prime}}$. So the measure of all those graphs is bounded above by

$$
\sum_{\substack{V^{\prime} \subseteq V \\\left|V^{\prime}\right|=k}} \sum_{\substack{E^{\prime} \subseteq\left[V^{\prime}\right]^{2} \\\left|E^{\prime}\right|=2 k}} \mu\left(\mathscr{G}_{E^{\prime}}\right)
$$

The crucial component in this sum is the summation over all subsets of size $k$; this will be estimated by a factor $N^{k}$; the other summations will be replaced by factors that depend on $k$ only. Altogether, we get an upper bound

$$
N^{k}\left(k^{2}\right)^{2 k}\left(4 N^{-1+\varepsilon}\right)^{2 k}=(2 k)^{4 k} N^{k} N^{-2 k(1-\varepsilon)}=(2 k)^{4 k} N^{k(2 \varepsilon-1)} \approx N^{k(2 \varepsilon-1)} .
$$


Now summing over all $k \leqslant M$ yields

$$
\sum_{k=1}^{M} N^{k(2 \varepsilon-1)} \leqslant M \cdot N^{2 \varepsilon-1}<1 / 10,
$$

as $M \ll_{\varepsilon} N$. Hence, the set of graphs satisfying the conclusion has measure $>0$, so it is nonempty.

Lemma 2.4. Let $0<\varepsilon<\frac{1}{2}$. There is an increasing sequence $\left\langle N_{\ell}: \ell \in \mathbb{N}\right\rangle$ of natural numbers and a sequence $\left\langle\left(V_{\ell}, E_{\ell}\right): \ell \in \mathbb{N}\right\rangle$ of graphs such that the following hold:

(1) $\max \left\{N_{\ell-1}^{2}+1,2^{3 N_{\ell-1}}, 1+\left|E_{\ell-1}\right|\right\}<N_{\ell}$.

(2) $V_{\ell}=\left[N_{\ell-1}, N_{\ell}\right)$.

(3) $\left|E_{\ell}\right| \geq N_{\ell}^{1+\varepsilon}$.

(4) For all $k \leqslant 2^{\ell+1} N_{\ell-1}$, the graph $\left(V_{\ell}, E_{\ell}\right)$ is $(k, 2 k)$-sparse.

Proof. We can choose $N_{\ell}$ by recursion; given $N_{\ell-1}$, Lemma 2.3 tells us how large $N_{\ell}$ has to be. In more detail, let $\varepsilon^{\prime}$ be such that $\varepsilon<\varepsilon^{\prime}<\frac{1}{2}$. Then by Lemma 2.3, there exist $N_{\ell}^{\prime}$ and a graph $\mathcal{G}$ with $N_{\ell}^{\prime}$ vertices and more than $\left(N_{\ell}^{\prime}\right)^{1+\varepsilon^{\prime}}$ edges which is $(k, 2 k)$-sparse for all $k \leq 2^{\ell+1} N_{\ell-1}$. Enlarging $N_{\ell}^{\prime}$ if necessary, we may assume that

- (1) holds (more precisely, $N_{\ell}^{\prime}$ is larger than the left hand side of (1)), and

- $(1+\varepsilon) \ln (2)<\left(\varepsilon^{\prime}-\varepsilon\right) \ln \left(N_{\ell}^{\prime}\right)$ and $2 N_{\ell-1} \leq N_{\ell}^{\prime}$.

Take $N_{\ell}:=N_{\ell-1}+N_{\ell}^{\prime}$. Let $\mathcal{G}_{\ell}$ be an isomorphic copy of $\mathcal{G}$ with $V_{\ell}=\left[N_{\ell-1}, N_{\ell}\right)$. Now (2) and (4) of the statement clearly hold for $\mathcal{G}_{\ell}$. To check (3), it is enough to show that $N_{\ell}^{1+\varepsilon} \leq\left(N_{\ell}^{\prime}\right)^{1+\varepsilon^{\prime}}$, that is,

$$
\ln \left(N_{\ell}^{1+\varepsilon}\right) \leq \ln \left(\left(N_{\ell}^{\prime}\right)^{1+\varepsilon^{\prime}}\right) .
$$

The following calculation proves $(*)$ :

$$
\begin{aligned}
\ln \left(N_{\ell}^{1+\varepsilon}\right) & =(1+\varepsilon) \ln \left(N_{\ell-1}+N_{\ell}^{\prime}\right) \leq(1+\varepsilon) \ln \left(2 N_{\ell}^{\prime}\right) \\
& =(1+\varepsilon) \ln \left(N_{\ell}^{\prime}\right)+(1+\varepsilon) \ln (2) \leq(1+\varepsilon) \ln \left(N_{\ell}^{\prime}\right)+\left(\varepsilon^{\prime}-\varepsilon\right) \ln \left(N_{\ell}^{\prime}\right) \\
& =\left(1+\varepsilon^{\prime}\right) \ln \left(N_{\ell}^{\prime}\right)=\ln \left(\left(N_{\ell}^{\prime}\right)^{1+\varepsilon}\right) .
\end{aligned}
$$

So our graphs $\left(V_{\ell}, E_{\ell}\right)$ have "many edges" on a large scale (i.e., looking at the whole graph), but only "few edges" on a small scale (looking at small induced subgraphs).

Definition 2.5. A $d$-ary (partial) function $f: V^{d} \rightarrow \mathbb{N}$ is defined to be $(k, l)$ modest iff for any $U_{0}, \ldots, U_{d-1} \subseteq V$ of size at most $k, f \uparrow\left(U_{0} \times \cdots \times U_{d-1}\right)$ has at most $l$ values.

Lemma 2.6. Let $(V, E)$ be a graph which is $(k, 2 k)$-sparse for all $k \leqslant M$. Let $f: V \times V \rightarrow \mathbb{N}$ be a symmetric function which takes different values on all edges in $E$ and is constantly zero outside $E$. Then $f$ has at least $|E|$ values but is $(k, 5 k)$-modest for all $k \leqslant M / 2$. 
Proof. For each $U_{1}, U_{2} \subseteq V$ of size $k \leqslant M / 2, E \cap\left(U_{1} \cup U_{2}\right)^{2}$ has at most $2 \cdot 2 k$ edges, so $f$ can take at most $4 k+1$ values on $U_{1} \times U_{2} \subseteq\left(U_{1} \cup U_{2}\right)^{2}$.

Corollary 2.7. There is an increasing sequence $\left\langle N_{\ell}: \ell \in \mathbb{N}\right\rangle$ of natural numbers and a sequence $\left\langle s_{\ell}: \ell \in \mathbb{N}\right\rangle$ of operations $s_{\ell}:\left[N_{\ell-1}, N_{\ell}\right)^{2} \rightarrow \mathbb{N}$ satisfying the following:

(1) $\max \left\{N_{\ell}^{2}+1,2^{3 N_{\ell}}, 1+\left|E_{\ell}\right|\right\}<N_{\ell+1}$.

(2) Each $s_{\ell}$ is $(k, 5 k)$-modest for all $k \leqslant 2^{\ell} N_{\ell-1}$.

(3) Each $s_{\ell}$ is $(k, 5 k)$-modest for all $k \geq N_{\ell+1}$.

(4) For all $\ell$, the range of $s_{\ell}$ has more than $N_{\ell}^{4 / 3}$ elements.

Proof. Let $\varepsilon=\frac{1}{3}$ and let $\left\langle N_{\ell}: \ell \in \mathbb{N}\right\rangle$ and $\left\langle\left(V_{\ell}, E_{\ell}\right): \ell \in \mathbb{N}\right\rangle$ be the sequences obtained from Lemma 2.4. In addition, for every $\ell \in \mathbb{N}$, let $s_{\ell}$ be the operation obtained from $\left(V_{\ell}, E_{\ell}\right)$ by Lemma 2.6. We claim that this choice satisfies the statement.

(1) follows from Lemma 2.4(1). Combining Lemma 2.4(4) with Lemma 2.6, one obtains (2). By Lemma 2.6, the range of $s_{\ell}$ has cardinality at most $\left|E_{\ell}\right|+1<N_{\ell+1}$. Hence, (3) holds trivially because of Lemma 2.4(1). Finally, (4) follows from Lemma 2.4(3) (combined with the choice of $\varepsilon$ and with Lemma 2.6).

From now on we fix sequences $\left\langle N_{\ell}: \ell \in \mathbb{N}\right\rangle$ and $\left\langle s_{\ell}: \ell \in \mathbb{N}\right\rangle$ as above.

Definition 2.8. For every $A \subseteq \mathbb{N}$, let $s_{A}: \mathbb{N} \times \mathbb{N} \rightarrow \mathbb{N}$ be defined from $s_{\ell}$ as follows: $s_{A}$ is $\bigcup_{\ell \in A} s_{\ell}$, extended by the value 0 wherever it is undefined (i.e., $s_{A} \uparrow\left[N_{\ell-1}, N_{\ell}\right) \times\left[N_{i-1}, N_{i}\right)$ is constantly zero for $\left.\ell \neq i\right)$.

\section{Lemma 2.9.}

(1) If $\ell<i$, then $s_{i}$ is $(k, 5 k)$-modest for all $k \leqslant 2^{\ell} N_{\ell}$.

(2) If $\ell \notin A$, then $s_{A}$ is $(k, 12 k)$-modest for all $k$ in $\left[N_{\ell}, 2^{\ell} N_{\ell}\right]$.

Proof. First we prove (1). By Lemma $2.7(2), s_{i}$ is $(k, 5 k)$-modest for all $k \leqslant$ $2^{i} N_{i-1}$, so certainly also for all $k \leqslant 2^{\ell} N_{\ell}$.

Now we prove (2). Let $X, Y$ be sets of size $k$, with $k$ in $\left[N_{\ell}, 2^{\ell} N_{\ell}\right]$. Let $X_{-}=X \cap N_{\ell}, X_{+}=X \backslash X_{-}$, and define $Y_{-}, Y_{+}$similarly. We have

$$
s_{A}[X \times Y] \subseteq s_{A}\left[X_{-} \times Y_{-}\right] \cup s_{A}\left[X_{+} \times Y_{+}\right] \cup\{0\} .
$$

Because $\ell \notin A, s_{A}$ is constantly 0 on $\left(X_{-} \times Y_{-}\right) \backslash\left(N_{\ell-1} \times N_{\ell-1}\right)$. Hence, the first set has size at most $N_{\ell-1}^{2} \leqslant N_{\ell}-1 \leqslant k-1$.

To estimate the size of $s_{A}\left[X_{+} \times Y_{+}\right] \cup\{0\}$, we partition $X_{+}$as $X_{+}=\bigcup_{i>\ell} X_{i}$ with $X_{i}:=X_{+} \cap\left[N_{i}, N_{i+1}\right)$, similarly for $Y_{+}$.

We can find sets $X_{i}^{\prime}, Y_{i}^{\prime} \subseteq\left[N_{i}, N_{i+1}\right)$, both of size $q_{i}:=\max \left(\left|X_{i}\right|,\left|Y_{i}\right|\right)$, with $X_{i} \subseteq X_{i}^{\prime}$ and $Y_{i} \subseteq Y_{i}^{\prime}$. Note that $q_{i} \leqslant\left|X_{i}\right|+\left|Y_{i}\right|$, so $\sum_{i} q_{i} \leqslant 2 k$.

We have $s_{A}\left[X_{+} \times Y_{+}\right] \cup\{0\} \subseteq\{0\} \cup \bigcup_{i>\ell} s_{i}\left[X_{i}^{\prime} \times Y_{i}^{\prime}\right]$. By (1), the function $s_{i}$ is $\left(q_{i}, 5 q_{i}\right)$-modest, so the sets $s_{i}\left[X_{i}^{\prime} \times Y_{i}^{\prime}\right]$ are at most of size $5 q_{i}$. Hence, $s_{A}\left[X^{+} \times Y^{+}\right]$has size at most $11 k$. So $s_{A}[X \times Y]$ has size at most $12 k$. 
Definition 2.10. Let $A_{1}, \ldots, A_{n} \subseteq \mathbb{N}$. A (binary) term in the operations $s_{A_{1}}, \ldots, s_{A_{n}}$ is a formal expression involving (some of) the variables $x, y$, (some of) the operations $s_{A_{1}}, \ldots, s_{A_{n}}$, as well as any unary operations. (We trust the reader to supply a formal definition by induction.)

The depth of a term $\tau$ is defined inductively as follows:

- $x$ and $y$ have depth 0 .

- For any unary operation $u$, the depth of $u(\tau)$ is 1 more that the depth of $\tau$.

- Let $m$ be the maximum of the depths of $\tau_{1}$ and $\tau_{2}$. Then the depth of $s_{A_{i}}\left(\tau_{1}, \tau_{2}\right)$ is $m+1$.

Every term naturally induces a binary operation on $\mathbb{N}$. (Note that the same operation may be represented by different terms, even terms of different depths.)

Lemma 2.11. Let $\tau$ be a term in the operations $s_{A_{1}}, \ldots, s_{A_{n}}$ of depth $d$. Let $\ell>d \log _{2}(12)$ and assume $\ell \notin A_{1} \cup \cdots \cup A_{n}$. Then we have:

(1) The operation represented by $\tau$ is $\left(N_{\ell}, 12^{d} N_{\ell}\right)$-modest.

(2) In particular, $\tau$ cannot represent the operation $s_{\ell}$, or $s_{B}$ for any $B$ containing $\ell$.

Proof. We start to show (1) by induction on $d$ (or more precisely, on $\tau$ ).

If $\tau$ is $x$ or $y$, then this is trivial.

If $\tau=u\left(\tau_{1}\right)$, then again the range of $u\left(\tau_{1}\right)$ is not larger than the range of $\tau_{1}$. Assume $\tau=s_{A_{i}}\left(\tau_{1}, \tau_{2}\right)$, where the depths of $\tau_{1}$ and $\tau_{2}$ are at most $d$. Observe the following:

- Both $\tau_{1}$ and $\tau_{2}$ are $\left(N_{\ell}, 12^{d} N_{\ell}\right)$-modest by the inductive assumption.

- By Lemma $2.9(2), s_{A_{i}}$ is $\left(12^{d} N_{\ell}, 12 \cdot 12^{d} N_{\ell}\right)$-modest. (Recall that we have $d \log _{2}(12) \leqslant \ell$, so $\left.12^{d} N_{\ell} \leqslant 2^{\ell} N_{\ell}.\right)$

Now let $U_{1}, U_{2} \subseteq \mathbb{N}$ be two sets, both of size at most $N_{\ell}$. Then, according to the previous observation, the ranges of $\tau_{1} \mid U_{1}$ and $\tau_{2} \mid U_{2}$ have size at most $12^{d} N_{\ell}$. Hence, again by the previous observation, the cardinality of the range of $\tau\left\lceil U_{1} \times U_{2}\right.$ is at most $12 \cdot 12^{d} N_{\ell}=12^{d+1} N_{\ell}$, as desired.

Now we turn to prove (2). By assumption, $12^{d} \leqslant 2^{\ell} \leqslant 2^{N_{\ell-1}}$. By (1) of Corollary 2.7, we have $2^{N_{\ell-1}}<N_{\ell}^{\frac{1}{3}}$, so $12^{d} N_{\ell} \leqslant 2^{N_{\ell}} \cdot N_{\ell}<N_{\ell}^{\frac{4}{3}}$. Hence, by (1) of the present lemma, $\left|\operatorname{range}\left(\tau \mid N_{\ell} \times N_{\ell}\right)\right| \leq 12^{d} N_{\ell}<N_{\ell}^{4 / 3}$, while, according to Corollary 2.7 (4), we have $\left|\operatorname{range}\left(s_{B} \mid N_{\ell}\right)\right|>N_{\ell}^{4 / 3}$.

Corollary 2.12. Let $B, A_{1}, \ldots, A_{n}$ be pairwise distinct subsets of $\mathbb{N}$ such that $B \backslash\left(A_{1} \cup \cdots \cup A_{n}\right)$ is infinite. Then $s_{B} \notin\left\langle s_{A_{1}}, \ldots, s_{A_{n}}\right\rangle_{\mathscr{O}^{(1)}}$.

Proof. Assume, seeking a contradiction, that $s_{B} \in\left\langle s_{A_{1}}, \ldots, s_{A_{n}}\right\rangle_{\mathscr{O}(1)}$. Then there exists a term $\tau$ in $A_{1}, \ldots A_{n}$ representing $s_{B}$. Let $d$ be the depth of $\tau$. Then there exists $\ell \in B \backslash\left(A_{1} \cup \cdots \cup A_{n}\right)$ with $\ell>d \log _{2}(12)$. Then by Lemma 2.11(2), $\tau$ does not represent $s_{B}$. This contradiction completes the proof. 
Fact 2.13. There exists an independent family $\left(A_{r}: r \in \mathbb{R}\right)$ of $\mathfrak{c}$ subsets of $\mathbb{N}$. That is, for all disjoint finite subsets $I_{+}, I_{-} \subseteq \mathbb{R}$, the set

$$
\bigcap_{r \in I_{+}} A_{i} \cap \bigcap_{r \in I_{-}}\left(\mathbb{N} \backslash A_{i}\right)
$$

is nonempty and even infinite.

Proof. This is well known. For example, replacing the base set $\mathbb{N}$ by $\mathbb{Q}[x]$, the set of all polynomials with rational coefficients, we can take $A_{r}:=\{p(x) \in$ $\mathbb{Q}[x]: p(r)>0\}$.

Proof of Theorem 1.1. Choose an independent family $\left(A_{r}: r \in \mathbb{R}\right)$ of subsets of $\mathbb{N}$. Then for all finite $S \subseteq \mathbb{R}$ and all $r \in \mathbb{R} \backslash S$, the set $A_{r} \backslash \bigcup_{s \in S} A_{s}$ is infinite. By Corollary 2.12, $\left\{s_{A_{r}}: r \in \mathbb{R}\right\}$ is a family of operations independent over $\mathscr{O}^{(1)}$ : for any $r \in \mathbb{R}$, we have $s_{A_{r}} \notin\left\langle s_{A_{p}}: p \in \mathbb{R} \backslash\{r\}\right\rangle_{\mathscr{M} \cap \mathscr{O}^{(d)}}$. By Lemma 1.4, we are done.

\section{Higher arities}

According to Definition 1.2, we say that an operation $f: X^{d} \rightarrow X$ is modest iff there is some $k$ such that for all $N>1, f$ is $(N, k N)$-modest, i.e., the set $f\left[X_{1} \times \cdots \times X_{d}\right]$ has at most $k N$ elements whenever each set $X_{i} \subseteq X$ has at most $N$ elements. We call a clone $C$ modest if all operations in $C$ are modest.

As we already observed in Subsection 1.1, the set of all modest operations is a clone (the greatest modest clone) and all unary operations are modest, as are all operations with finite range.

This section is devoted to the second main result of the paper, which is Theorem 1.3. We postpone the proof of this theorem to the end of this section. The number $d$ will be fixed throughout this section.

In the previous section, we defined the notion of (binary) terms. For technical reasons, in the present section we need a more precise, and somewhat more general definition of terms. Throughout the present section, we use the word term in the sense of the following definition.

\section{Definition 3.1.}

- We fix a language with object variables $x_{i}$ for $i \in \mathbb{N}$ and formal operation variables $\mathrm{f}_{j}^{i}$ for $i, j \in \mathbb{N}$, where the superscript $i$ denotes the formal arity of $\mathrm{f}_{j}^{i}$. Terms are defined as usual: each object variable is a term, and whenever $t_{1}, \ldots, t_{i}$ are terms and $j \in \mathbb{N}$, then $\mathrm{f}_{j}^{i}\left(t_{1}, \ldots, t_{i}\right)$ is a term, as well.

- The set of all terms can be enumerated as $\left\{\tau_{1}, \tau_{2}, \ldots\right\}$ such that $\tau_{m}$ contains at most $m$ occurrences of operation symbols, and each operation symbol occurring in $\tau_{m}$ is at most $m$-ary.

- Let $\tau$ be a term. We say that a family of functions $\bar{g}=\left(g_{j}^{i}:(i, j) \in S\right)$ is suitable for $\tau$ iff each $g_{j}^{i}$ has arity $i$ and $(i, j) \in S$ whenever the variable $\mathrm{f}_{j}^{i}$ appears in $\tau$. 
- Let $\tau$ be a term and suppose $\bar{g}=\left(g_{j}^{i}:(i, j) \in S\right)$ is a family of operations on $X$ which is suitable for $\tau$. Then plugging in the $g_{j}^{i}$ for the $\mathrm{f}_{j}^{i}$ will yield an operation on $X$ which we denote by $\tau[\bar{g}]$.

Definition 3.2. Let $d \geq 2$. For any set $V$, we let $[V]^{d}$ be the set of $d$-element subsets of $V$. The structure $(V, E)$ is defined to be a d-uniform hypergraph iff $E \subseteq[V]^{d}$. The elements of $E$ are called the hyperedges of $(V, E)$.

Every $V^{\prime} \subseteq V$ naturally induces a hypergraph $\left(V^{\prime}, E \cap\left[V^{\prime}\right]^{d}\right)$, which we may also denote by $\left(V^{\prime}, E\left\lceil V^{\prime}\right)\right.$.

We say that $(V, E)$ is $(k, l)$-sparse iff for every $Z \subseteq V$ of size at most $k$, the hypergraph $(Z, E\lceil Z)$ has at most $l$ hyperedges.

Definition 3.3. The support of a partial function $f$ is the set of elements in the domain of $f$ where the value of $f$ is not equal to 0 .

Lemma 3.4. Fix $d, k, \varepsilon$. Let $V$ be a set of cardinality $N$ and let $(V, E)$ be $a(d+1)$-uniform hypergraph with at least $N^{d+\varepsilon}$ hyperedges. If $N$ is large enough, then there is an operation $s: V^{d+1} \rightarrow V$ whose support is contained in $E$ and whose values are in $\{0,1\}$ such that for any set $W$ with $V \subseteq W$ and $|W| \leq k N$, the following holds: whenever $\tau \in\left\{\tau_{1}, \ldots, \tau_{k}\right\}$, and $\overline{\bar{g}}=\left(g_{j}^{i}\right)_{i, j}$ is a suitable sequence of operations for $\tau$ on $W$ with each $g_{j}^{i}$ being

- either of arity at most d

- or of arity $d+1$ with support of size at most $3 N \log _{2} N$,

then $\tau[\bar{g}]$ does not represent $s$. In particular, there exists $e \in E$ such that $s$ and $\tau[\bar{g}]$ have different values on $e$.

If $N$ satisfies the above conditions, then we will say that $N$ is $k$-large.

Proof. Let $W$ be a set containing $V$ with $|W|=k N$. Clearly, it is enough to show that there exists an operation $s: V^{d+1} \rightarrow V$ satisfying the statement for this particular $W$. There are only $(k N)^{(k N)^{d}} d$-ary operations on $W$, and only $k$ terms to be considered. A support is a subset of $[W]^{d+1}$; there are fewer than $\left(\begin{array}{c}(k N)^{d+1} \\ 3 N \log _{2} N\end{array}\right) \leqslant(k N)^{3 N \log _{2}(N)(d+1)}$ possible supports of size $3 N \log _{2} N$. For any fixed support of size $3 N \log _{2} N$, there are at most $(k N)^{3 N \log _{2} N}$ possible operations that have this support. By the enumeration fixed in Definition 3.1, each term $\tau_{i}(i \leq k)$ contains at most $k$ many operation variables. Counting the possibilities of choosing $k$ many $d$-ary operations and $k$ many $(d+1)$-ary operations with support of size at most $3 N \log _{2} N$, one can see that altogether there are fewer than

$$
t:=(k N)^{(k N)^{d} \cdot k} \cdot k \cdot(k N)^{3 N \log _{2}(N)(d+1) k} \cdot(k N)^{3 N \log _{2}(N) k}
$$

operations represented by such terms. We may assume $k \leqslant \log _{2} N$. Estimating $k$ by $N$ or by $\log _{2} N$, one obtains

$$
t \leq\left(\log _{2} N\right) \cdot\left(N \cdot \log _{2} N\right)^{\log _{2} N \cdot\left(N \log _{2} N\right)^{d}} \cdot N^{6 N(d+1) \log _{2}^{2} N} \cdot N^{6 N \log _{2}^{2} N} .
$$

Recall that for any $\delta>0$ and $d \in \mathbb{N}$ and for large enough $N$, one has $\log _{2}^{d} N \leq$ $N^{\delta}$. Let $0<\delta<\varepsilon$. Then for large enough $N$, each of the four factors of $t$ 
can be estimated by $N^{\frac{1}{4} \cdot N^{d+\delta}}$. Consequently, for large enough $N$, we have $t<N^{N^{d+\delta}}=2^{N^{d+\delta} \cdot \log _{2} N}$. This number (for large enough $N$ ), is certainly less than $2^{N^{d+\varepsilon}}$.

But there are at least $2^{N^{d+\varepsilon}}$ possible operations on $E$ with values in $\{0,1\}$. So not all of them are representable.

Lemma 3.5. Let $0<\varepsilon<1 / 2$. Then there are sequences $\bar{N}=\left\langle N_{\ell}: \ell<\mathbb{N}\right\rangle$, $\bar{E}=\left\langle E_{\ell}: \ell<\mathbb{N}\right\rangle$ with the following properties:

(1) $\bar{N}$ is strictly increasing and in fact $N_{\ell-1}^{d+1}<N_{\ell}, 2^{\ell} \leq N_{\ell}$, and $N_{\ell}$ is $\ell$-large for all $\ell$. We will write $V_{\ell}$ for the interval $\left[N_{\ell-1}, N_{\ell}\right)$.

(2) $\left(V_{\ell}, E_{\ell}\right)$ is a $(d+1)$-uniform hypergraph with more than $N_{\ell}^{d+\varepsilon}$ hyperedges.

(3) For every $k \leqslant N_{\ell-1}^{2},\left(V_{\ell}, E_{\ell}\right)$ is $(k, 2 k)$-sparse.

Proof. This proof is only a slight variation of the proof of Lemma 2.7, so we will be brief.

Assume $N_{\ell-1}$ has already been defined. We will choose $N_{\ell}$ after a certain amount of extra work such that $N_{\ell} \gg N_{\ell-1}$. Assume, for a moment, that $N_{\ell}$ is already defined. Let $V_{\ell}:=\left[N_{\ell-1}, N_{\ell}\right)$. Let $J$ be the cardinality of the set $\left[V_{\ell}\right]^{d+1}$ of all potential hyperedges: $J=\left(\begin{array}{c}N_{\ell}-N_{\ell-1} \\ d+1\end{array}\right)$.

On the set of all $(d+1)$-uniform hypergraphs (which we may identify with $2^{J}$ ), we define a product measure by declaring the probability of each potential hyperedge to be $p:=2(d+1) ! \cdot N^{\varepsilon-1}$.

So the expected number of hyperedges of a random hypergraph is $p J=$ $2(d+1) ! \cdot N_{\ell}^{\varepsilon-1} \cdot\left(\begin{array}{c}N_{\ell}-N_{\ell-1} \\ d+1\end{array}\right) \approx 2 N_{\ell}^{\varepsilon-1} \cdot N_{\ell}^{d+1}=2 N_{\ell}^{d+\varepsilon}$. Again using Chebyshev's inequality, we see that with high probability a random hypergraph will have more than $N_{\ell}^{d+\varepsilon}$ hyperedges.

Now we estimate the probability that there is a sub-hypergraph with $k \leqslant$ $N_{\ell-1}^{2}$ vertices which has more than $2 k$ hyperedges, and we will show that it is very low.

For each potential $k$, there are at most $\left(\begin{array}{c}N_{\ell} \\ k\end{array}\right) \leqslant N_{\ell}^{k}$ subsets; for each such subset $S$, the probability that a given set $H$ of hyperedges with $j:=|H| \geq 2 k$ appears as a subset of $E\left\lceil S\right.$ is $\leqslant p^{j} \leqslant p^{2 k}$. There are $\left(\begin{array}{c}k^{d} \\ j\end{array}\right) \leqslant 2^{k^{d}}$ possibilities for $H$. So the probability that such a bad subgraph of size $k$ exists is bounded from above by $N_{\ell}^{k} \cdot p^{2 k} \cdot 2^{k^{d}}$. There are $N_{\ell-1}^{2}$ possibilities for $k$, so we have to choose $N_{\ell}$ such that

$$
\sum_{k=1}^{N_{\ell-1}^{2}} N_{\ell}^{k} \cdot p^{2 k} 2^{k^{d}} \leq \frac{1}{2} .
$$

But $N_{\ell}^{k} \cdot p^{2 k} \approx N_{\ell}^{k} N_{\ell}^{(\varepsilon-1) 2 k}=N_{\ell}^{k(2 \varepsilon-1)}$ which converges to 0 if $N_{\ell}$ converges to infinity. Hence, one may choose $N_{\ell}$ so large, that

$$
N_{\ell}^{k} \cdot p^{2 k}<\frac{1}{N_{\ell-1}^{2} \cdot 2^{\left(N_{\ell-1}^{2}\right)^{d}}}
$$


and $N_{\ell}>\max \left\{2^{\ell}, N_{\ell-1}^{d+1}\right\}$ hold. Further increasing $N_{\ell}$ if necessary, we may choose it to be $\ell$-large, as well. Estimating $2^{k^{d}}$ by $2^{\left(N_{\ell-1}^{2}\right)^{d}}$ in the left hand side of $(* *)$, it follows that the inequality in $(* *)$ holds.

So the set of hypergraphs on $V_{\ell}$ which are not $(k, 2 k)$-sparse for some $k \leq$ $N_{\ell-1}^{2}$ has measure at most $\frac{1}{2}$, while almost all hypergraphs on $V_{\ell}$ have $N_{\ell}^{d+\varepsilon}$ hyperedges. It follows that there exist $N_{\ell}$ and $E_{\ell}$ satisfying the requirements of the lemma, and thus, the sequences in the statement can be constructed recursively.

Definition 3.6. Let $\bar{N}$ and $\bar{E}$ be as in Lemma 3.5. For each $V_{\ell}=\left[N_{\ell-1}, N_{\ell}\right)$, let $s_{\ell}$ be a $(d+1)$-ary operation with support $E_{\ell}$ which differs on $E_{\ell}$ from each $\tau_{i}[g](i \leqslant \ell, \bar{g}$ as in Lemma 3.4).

For each infinite $A \subseteq \mathbb{N}$, let $s_{A}:=\bigcup_{\ell \in A} s_{\ell}$ (where we replace all undefined values of $s_{A}$ with 0 ).

Lemma 3.7. Let $B \subseteq \mathbb{N}$ be infinite and assume $\ell \in \mathbb{N} \backslash B$. Let $W \subseteq \mathbb{N}$ be such that $|W| \leq \ell \cdot N_{\ell}$. Then the cardinality of the support of $s_{B} \mid W^{d+1}$ is at most $N_{\ell}\left(1+2 \log _{2} N_{\ell}\right)$.

Proof. Throughout this proof, we write $\operatorname{supp}(f)$ for the support of a function $f$. Let $W_{1}=W \cap\left[0, N_{\ell-1}\right), W_{2}=W \cap\left[N_{\ell-1}, N_{\ell}\right)$, and $W_{3}=W \backslash\left(W_{1} \cup W_{2}\right)$. By construction,

$$
\operatorname{supp}\left(s _ { B } \lceil W ^ { d + 1 } ) \subseteq \operatorname { s u p p } \left(s _ { B } \lceil W _ { 1 } ^ { d + 1 } ) \cup \operatorname { s u p p } \left(s _ { B } \lceil W _ { 2 } ^ { d + 1 } ) \cup \operatorname { s u p p } \left(s_{B}\left\lceil W_{3}^{d+1}\right) .\right.\right.\right.\right.
$$

Clearly, $\left|\operatorname{supp}\left(s_{B} \mid W_{1}^{d+1}\right)\right| \leq N_{\ell-1}^{d+1}$ and $N_{\ell-1}^{d+1} \leq N_{\ell}$ by Lemma 3.5(1). In addition, $\operatorname{supp}\left(s_{B}\left\lceil W_{2}^{d+1}\right)\right.$ is empty because $\ell \notin B$. Clearly,

$$
\left|W_{3}\right| \leq|W| \leq \ell \cdot N_{\ell} \leq \log _{2}\left(N_{\ell}\right) N_{\ell}
$$

(in the last estimation, we used Lemma $3.5(1): \ell \leq \log _{2} N_{\ell}$ ). In addition, by Lemma 3.5 (3), for any $j>\ell,\left(V_{j}, E_{j}\right)$ is $\left(N_{\ell} \log _{2} N_{\ell}, 2 N_{\ell} \log _{2} N_{\ell}\right)$-sparse. It follows that $\left|\operatorname{supp}\left(s_{B} \mid W_{3}^{d+1}\right)\right| \leq 2 N_{\ell} \log _{2} N_{\ell}$. Combining these observations, the statement follows.

Lemma 3.8. If $f_{1}, \ldots, f_{m}$ are $\left(k, k^{\prime}\right)$-modest $d$-ary operations and $g$ is a $\left(k^{\prime}, k^{\prime \prime}\right)$-modest $m$-ary operation, then $g\left(f_{1}, \ldots, f_{m}\right)$ is $\left(k, k^{\prime \prime}\right)$-modest.

Proof. The proof is easy.

Lemma 3.9. Let $\mathscr{M}$ be the clone of all modest operations. Let $A \backslash\left(B_{1} \cup \cdots B_{r}\right)$ be infinite. Then $s_{A} \notin\left\langle s_{B_{1}}, \ldots, s_{B_{r}}\right\rangle \mathscr{M \cap O}^{(d)}$.

Proof. For any term $\tau$ and any suitable sequence $\bar{g}$ (consisting only of operations in $\left.\left\langle\left(\mathscr{M} \cap \mathscr{O}^{(d)}\right) \cup\left\{s_{B_{1}}, \ldots, s_{B_{r}}\right\}\right\rangle\right)$, we will find $\ell \in A$ such that $\tau[g]$ disagrees with $s_{\ell}$ (hence also with $s_{A}$ ) on $E_{\ell}$.

So fix a term $\tau=\tau_{i}$ and $\bar{g}$. Let $\nu$ be the number of subterms of $\tau$ and let $k$ witness that all operations in $\bar{g}$ are modest. Let $\ell>\nu \cdot k^{i}$ be in $A \backslash\left(B_{1} \cup \cdots B_{r}\right)$. We claim that for each subterm $\sigma$ of $\tau$ (of depth $s$ ), the range of $\sigma[\bar{g}]$ over the domain $V_{\ell}^{d+1}$ has cardinality at most $N_{\ell} \cdot k^{s}$. 
This can be proved by induction on the depth of $\sigma$ using Lemma 3.8 combined with the fact that the operations $s_{B_{j}}$ take only 2 values, and that all other operations in $\bar{g}$ are modest, witnessed by $k$.

Recall that according to the enumeration fixed in Definition 3.1, the depth of $\tau=\tau_{i}$ is at most $i$. So the set of all intermediate values in the computation of $\tau[g]$ on $E_{\ell}$ has size at most $\nu \cdot k^{i} N_{\ell}<\ell N_{\ell}$. Let $W \supseteq V_{\ell}$ be a set of size at most $\ell N_{\ell}$ containing $\{0,1\}$ and all these intermediate values. The term $\tau$ induces a partial function $\tau[\bar{g}]\left\lceil E_{\ell}\right.$. By replacing all values of the operations in $\bar{g}$ by 0 if they are outside $W$, we get a sequence $\bar{g}^{\prime}$ of operations with the following properties:

- $\tau\left[\bar{g}^{\prime}\right]$ is a total function from $W^{d+1}$ to $W$.

- $\tau\left[\bar{g}^{\prime}\right]$ agrees with $\tau[\bar{g}]$ on $E_{\ell}$.

- All operations in $\bar{g}^{\prime}$ are either some $s_{B_{j}}$ or an operation of arity at most $d$.

By Lemma 3.7, the support of each $s_{B_{j}} \mid W^{d+1}$ is at most $N_{\ell}\left(1+2 \log _{2} N_{\ell}\right) \leq$ $3 N_{\ell} \log _{2} N_{\ell}$. So by the construction of $s_{\ell}$, and by Lemma $3.4, s_{\ell}$ disagrees with $\tau\left[\bar{g}^{\prime}\right]$ somewhere on $E_{\ell}$; so $s_{\ell}$ also disagrees with $\tau[\bar{g}]$.

Now we are ready to prove Theorem 1.3.

Proof of Theorem 1.3. Similarly to the proof of Theorem 1.1, choose an independent family $\left(A_{r}: r \in \mathbb{R}\right)$ of subsets of $\mathbb{N}$. Then for all finite $S \subseteq \mathbb{R}$ and all $r \in \mathbb{R} \backslash S$, the set $A_{r} \backslash \bigcup_{s \in S} A_{s}$ is infinite. By Lemma 3.9, $\left\{s_{A_{r}}: r \in \mathbb{R}\right\}$ is a family of operations independent over $\mathscr{M} \cap \mathscr{O}^{(d)}$ : for any $r \in \mathbb{R}$, we have $s_{A_{r}} \notin\left\langle s_{A_{p}}: p \in \mathbb{R} \backslash\{r\}\right\rangle_{\mathscr{O}^{(1)}}$. By Lemma 1.4, we are done.

Corollary 3.10. There exists a clone $C$ on $\mathbb{N}$ such that for any $d \in \mathbb{N}$, there are $2^{\mathfrak{c}}$ clones $D$ with $C \cap \mathscr{O}^{(d)}=D \cap \mathscr{O}^{(d)}$.

Proof. Let $C$ be the clone generated by all operations whose ranges are a subset of $\{0,1\}$. To check that this $C$ satisfies the statement of the corollary, let $d \in \mathbb{N}$ and let $C^{\prime}$ be the clone generated by all at most $d$-ary operations whose ranges are contained in $\{0,1\}$. Then $C \cap \mathscr{O}^{(d)}=C^{\prime} \cap \mathscr{O}^{(d)}$ and $C^{\prime}$ is modest. Therefore, by Theorem 1.3 , there exist $2^{\mathfrak{c}}$ many clones $D$ with $D \cap \mathscr{O}^{(d)}=C^{\prime} \cap \mathscr{O}^{(d)}=C \cap \mathscr{O}^{(d)}$.

\section{REFERENCES}

[1] Gavrilov, G.P.: On functional completeness in countably-valued logic. Problemy Kibernet. 15, 5-64 (1965)

[2] Goldstern, M.: Analytic clones. http://arxiv.org/math.RA/0404214. (2004)

[3] Goldstern, M., Pinsker, M.: A survey of clones on infinite sets. Algebra Universalis 59, 365-403 (2008)

[4] Goldstern, M., Shelah, S.: Clones on regular cardinals. Fund. Math. 173, 1-20 (2002)

[5] Machida, H.: The clone space as a metric space. Acta Appl. Math. 52, 297-304 (1998)

[6] Pinsker, M.: Monoidal intervals of clones on infinite sets. Discrete Math. 308, 59-70 (2008) 
MARTin GoldSTERN

Algebra/DMG, TU Wien, Wiedner Hauptstr 8-10/104, 1040 Wien, Austria

e-mail: goldstern@tuwien.ac.at

$U R L:$ http://www.tuwien.ac.at/goldstern/

\section{GÁbor SÁGI}

BUTE Department of Algebra and Alfréd Rényi Institute of Mathematics, Hungarian Academy of Sciences, Reáltanoda u. 13-15, 1053 Budapest, Hungary

e-mail: sagi@renyi.hu

\section{SAHARON Shelah}

Einstein Institute of Mathematics, Edmond J. Safra Campus, Givat Ram, The Hebrew University of Jerusalem, Jerusalem, 91904, Israel, and Department of Mathematics,

Rutgers University, New Brunswick, NJ 08854, USA

e-mail: shelah@math.huji.ac.il

URL: http://shelah.logic.at/ 